\title{
COMPUTER STUDY OF NONEQUILIBRIUM EXCITATION IN RECOMBINING NITROGEN PLASMA NOZZLE FLOWS $\dagger$
}

\author{
Stuart $\mathbf{W}$. Bowen* \\ The University of Michigan, Ann Arbor, Michigan \\ Chul Park** \\ NASA Ames Research Center, Moffett Field, California
}

\begin{abstract}
The nonequilibrium neutral atom excited state densities, electron densities, electron and heavy particle temperatures are computed for a fully dissociatsd partially ionized nitrogen plasma expanding in a nozzle starting from equilibrium in the settling chamber. The degree of excitation non equiliorium at the exit of a convergent-divergent nozzle having an area ratio of 22 is systematically investigated for chamber pressures between 0.01 and 1,000 atm and chamber temperatures between 6,000 and $18,000^{\circ} \mathrm{K}$. Thermal nonequilibrium at the exit rises to a maximum in the chamber pressure range between 1 and $10 \mathrm{~atm}$, while excitation nonequilibrium is smallest in this pressure range, due to the competing effects of the three-body recombination and collisional coupling terms in the electron energy equation. Increasing chamber pressure above $10 \mathrm{~atm}$ produces increasingly severe and unexpected departure from excitation equilibrium.
\end{abstract}

\section{List of Svmbols}

\section{A}

$A(i, j)$

$\vec{B},\left(B_{i j}\right)$

$\stackrel{b_{2}}{\vec{c}}-\mathrm{b}_{6}$

$\overrightarrow{\mathrm{D}},\left(\mathrm{D}_{\mathrm{i}}\right)$

$\mathbf{E}_{\infty}$

$\mathbf{E}(\mathbf{i})$

g(i)

$\mathrm{H}$

$\mathrm{h}$

$\mathbf{i}, \mathbf{j}$

$\mathbf{K}(\mathbf{i}, \mathbf{j})$

$\mathbf{k}$

$\mathbf{k}_{\mathbf{R}}$

$\mathrm{m}_{\mathrm{e}}, \mathrm{m}_{\mathrm{h}}$

$\dot{\mathrm{m}}$ cross-sectional area of nozzle

radiative transition rate

matrix whose elements are $B_{i j}$ in

Eq. (4)

constants specifying the nozzle shape

vector whose elements are $C_{i}$ in

Eq. (4)

vector whose elements are $D_{i}$ in

Eq. (4)

ionization potential

energy level of ith state measured

from ground state

statistical weight of ith state

enthalpy, see Eq. (14)

Planck constant

dummy indices

collisional transition rate coefficient

Boltzmann constant

ionic recombination rate

electron and heavy particle mass, respectively

mass flow rate through nozzle

\begin{tabular}{|c|c|}
\hline $\mathbf{N}(\mathbf{i})$ & population of ith state \\
\hline $\mathrm{N}_{2}, \mathrm{Ne}_{\mathrm{e}}$ & atom and electron density \\
\hline $\mathrm{N}_{\mathrm{aE}}$ & atom density under equilibrium \\
\hline$N_{E}(i)$ & lation of ith state under equilibrium \\
\hline $\mathrm{Ne}$, coll & $\begin{array}{l}\text { rate of change of electron density by } \\
\text { collisional process }\end{array}$ \\
\hline$\dot{\mathrm{N}}_{e}, \mathrm{rad}$ & $\begin{array}{l}\text { rate of change of electron density by } \\
\text { radiative process }\end{array}$ \\
\hline $\mathbf{N}_{\mathrm{h}}$ & heavy particle density $=N_{a}+N_{e}$ \\
\hline & \\
\hline Q rad & e power loss \\
\hline & adius \\
\hline 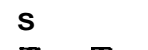 & constant specifying pressure variation \\
\hline $\mathrm{U}_{\mathrm{U}}^{\mathrm{T}}, \mathrm{T}_{\mathrm{h}}$ & $\begin{array}{l}\text { eleetron and heavy particle temperatur } \\
\text { plasma velocity }\end{array}$ \\
\hline$\nabla_{\mathrm{e}}$ & electron thermal velocity \\
\hline & $\mathrm{di}$ \\
\hline $\mathrm{Z}_{\mathrm{a}}, \mathrm{Z}_{+}$ & atom and ion partition function \\
\hline$\alpha$ & $=\mathrm{N}_{\mathrm{e}} / \mathrm{N}_{\mathrm{h}}$ \\
\hline$\alpha_{\mathbf{r}}$ & \\
\hline$\nu_{\mathrm{eh}}$ & $\begin{array}{l}\text { electron-heavy particle collision } \\
\text { frequency }\end{array}$ \\
\hline X & $\begin{array}{l}\text { chemical and excitation nonequilibrium } \\
\text { parameter in Eq. (7) }\end{array}$ \\
\hline & nensionless pol \\
\hline
\end{tabular}

Subscript

a

characteristic value

cham settling chamber

E equilibrium value required by the local nonequilibrium $\mathrm{T}_{e}$ and $\mathrm{N}_{e}$

EQ complete equilibrium value

$e \quad$ electron

h heavy particle

* throat

$+\quad$ ion

\section{Introduction}

Previous studies of electron-ion recombination in expanding plasma flows $1,2,3$ have shown that significant departures from equilibrium can be expected in typical wind tunnel nozzles in the fol lowing three ways;

†Work performed at the Ames Research Center under a cooperative program between the University of Santa Clara and Ames Research Center while the senior author was a Visiting Assistant Professor, University of Santa Clara, Santa Clara, California.

*Assistant Professor, Department of Aerospace Engineering.

**Research Scientist 
1. the electrons and heavy particles are at different temperatures (thermal nonequilibrium),

2. the electron density does not satisfy the Saha equation (chemical nonequilibrium), and

3. the bound electronic states of the atoms are not populated according to the Boltzmann distribution law (excitation nonequilibrium).

Whether or not any of these deviations from equilibrium are of importance in practice depends, of course, on the application or use to which the fllow is put. For example, the excited population distributions are crucial for spectroscopic diagnosis of plasma flow and the possible generation of stimulated emission, although they are of little consequence to aerodynamic forces and moments.

Although the fundamental physical principles that govern the relaxation of these nonequilibrium phenomena are known, the effects of the inter coupling between them are not well understood, particularly in a high speed fluid flow. The purpose of the present study is to systematically investigate the consequences of the intercoupling among the three nonequilibrium processes and the fluid conservation equations, by numerical $\mathrm{com}^{-}$ putation. An ionically recombining flow of nitrogen expanding through a converging-diverging nozzle is studied, starting from equilibrium stagnation conditions. The nitrogen plasma is assumed to be fully dissociated and partially ionized throughout the nozzle. The evolution of the nonequilibrium flow properties have been systematically calculated as functions of the chamber pressure and temperature. The study focuses on the unusual interaction between the thermal and chemical nonequilibrium, and also on the very large effects these two nonequilibrium phenomena have upon the atomic excited state distributions.

\section{Derivation of Equations}

\section{Assumptions}

In order to simplify the analysis, the following assumptions are made:

(i) The electrons and heavy particles each have a Maxwellian velocity distribution at temperatures $\mathrm{T}_{e}$ and $\mathrm{T}_{h}$, respectively, since the self-collision times for each species are very much smaller than other characteristic times. As a corollary, the continuum flow concept is assumed to hold.

(ii) The flow is steady and quasi-one dimensional, radial variations being neglected.

(iii) Thereare noimposed electric and magnetic fields. As a result, the electrons and lons have a common directed velocity.

(iv) Viscous diffusion and thermal conduction effects are neglected.

(v) The plasma is neutral and consists of neutral atoms, singly charged ions and electrons. Molecular recombination is neglected .

(vi) The radiative power loss is approximated by a simple expression, see Eq. (15).

\section{Electronic State Population}

Because diffusion is neglected in (iv) above, the rate of change of the electronic state population of the ith state, $\mathrm{N}(\mathrm{i})$, is controlled only by the chemical rates; that is, it is the difference between the sum of the rates of all incoming electronic transitions and that of all outgoing transitions 4,5 ,

$$
\begin{gathered}
\rho u \frac{d}{d x}\left[\frac{N(i)}{N_{h}}\right]=\left[-m_{h} N(i)\left\{\sum_{\substack{j=1 \\
j \neq i}}^{\infty} K(i, j)+K(i, \infty)\right.\right. \\
\left.+\frac{1}{N_{e}} \sum_{j=1}^{i-1} A(i, j)\right\}+m_{h} \sum_{\substack{j=1 \\
j \neq i}}^{\infty} N(j)\{K(j, i \\
\left.\left.+\frac{A(j, i)}{N_{e}}\right\}+m_{h} N_{e}\{K(\infty, i)+A(\infty, i)\}\right] N_{e}
\end{gathered}
$$

Here, $\mathbf{x}$ is the distance along the nozzle, $\rho$ and $u$ are the density and velocity of the plasma, $m_{h}$ and $\mathrm{N}_{\mathrm{h}}$ are the mass and the number density of the heavy particle, respectively, and $\mathrm{N}_{\mathrm{e}}$ is the electron density. $K(i, j)$ is the coefficient of the rate of electronic transitions of an atom from an initial state $i$ to a final state $j$ as a result of a collision with an electron. The state corepresents the ionized state; thus $K(i, \infty)$ and $K(\infty, i)$ denote the collisional ionization and recombination rate coefficients. Likewise, $A(i, j)$ is the electronic rate coefficient for radiative transition from an initial (upper) state $i$ to a final (lower) state $\mathbf{j}, A(\infty, i)$ being the radiative recombination rate coefficient. In addition, there is a relationship connecting $\mathrm{N}(\mathrm{i})$ 's to the atom density $\mathrm{N}_{\mathrm{a}}$,

$$
N_{a}=\sum_{i=1}^{\infty} N(i)
$$

It is convenient to express the electronic state pop:slation $\mathrm{N}(\mathrm{i})$ in terms of the dimensionless quantity $\psi(\mathrm{i})$

$$
\psi(i)=N(i) / N_{E}(i)
$$

where $N_{E}(i)$ is the equilibrium population required 
by the local electron density and temperature

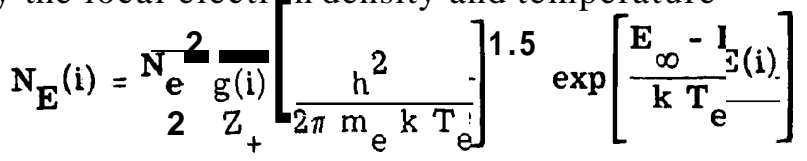

Here, $g(\mathbf{i})$ and $\mathrm{Z}$ are the statistical weight of the ith state and the ion partition function, respectively, $\mathbf{h}$ is the Planck constant, $\mathbf{m}_{\mathbf{e}}$ the electron mass, $\mathbf{E}(\mathbf{i})$ the energy level of the ith state measured from the ground state and $E_{\infty}$ is the ionization potential. In terms of the dimensionless population $\psi($ i), Eq. (1)and (2) can be combined symbol ically ${ }^{5}$ in the form

$$
\overrightarrow{\mathbf{B}} \cdot \vec{\psi}=\overrightarrow{\mathrm{C}}+x \overrightarrow{\mathrm{D}}
$$
where $\vec{B}$ is the matrix whose elements $B_{i j}$ are
functions only of $T_{e}$ and $N_{e}$ as follows;

For

$$
\mathrm{i}=1 \text {, }
$$

$$
\mathbf{B}_{1 \mathbf{j}}=\mathbf{N}_{\mathbf{E}}(\mathbf{j}) / \mathbf{N}_{\mathbf{E}}{ }^{2}\left(=\text { function of } \mathrm{T}_{\mathrm{e}} \text { alone }\right)
$$

For

$$
\mathrm{i}>1, \mathrm{j}=\mathrm{i} \text {, }
$$

$$
B_{11}=-\sum_{\substack{j=1 \\ j \neq i}}^{\infty} K(i, j)-K(i, \infty)-\frac{1}{N_{e}} \sum_{j=1}^{i-1} A(i, j)
$$

For

$$
\text { i }>1, \text { j f i, }
$$

$$
B_{i j}=K(i, j)+\frac{N_{E}(j)}{N_{E} W} \frac{A(j, i)}{N_{e}}
$$

$\vec{\psi}$ is a vector with component $\psi(\mathrm{i})$, and $\overrightarrow{\mathrm{D}}$ is a vector whose components $D_{i}$ 's are all zero except $D_{1}$ which is

$$
\mathrm{D}_{1}=\mathrm{N}_{\mathrm{aE}} / \mathrm{N}_{\mathrm{e}}^{2} \text { (= function of } \mathrm{T}_{\mathrm{e}} \text { alone) }
$$

where $\mathrm{N}_{a E}$ is the equilibrium atom density required by the actual electron density and temperature, i.e. Saha equilibrium value

$$
\mathrm{N}_{\mathrm{aE}}=\frac{\mathrm{N}_{\mathrm{e}}^{2}}{2} \frac{\mathrm{a}}{\mathrm{Z}_{+}}\left[\frac{\mathrm{h}^{2}}{2 \pi \mathrm{m}_{\mathrm{e}} \mathrm{kT} \mathrm{T}_{\mathrm{e}}}\right]^{1.5} \exp \left[\begin{array}{c}
\frac{\mathrm{E}}{\infty} \\
\mathrm{kT}
\end{array}\right] \text { (5) }
$$

in which $\mathrm{Za}$ is the atom partition function. $\overrightarrow{\mathrm{C}}$ is a vector with component $C_{i}$ which is zero for $i=1$, and for $\mathrm{i}>1$,

$$
\begin{gathered}
C_{i}=-K(i, \infty)-\frac{N_{e}}{N_{E}(i)} A(\infty, i)+\frac{\psi(i)}{N_{e}} u \frac{d}{d x}\{\ln \psi(i)\} \\
i>1
\end{gathered}
$$

Also $x$ is the ratio

$$
\left.X=N_{a} / N_{a E} \text { (= function of } N_{a}, N_{e} \text { and } T_{e}\right)(7)
$$

which is a measure of the degree of chemical nonequinitisitiom.
When the property of the plasma varies slowly along the nozzle, one can neglect the last term in Eq. (6) in comparison with the preceding two terms in the same equation. When such conditions exist, Eq. (4) becomes a system of algebraic equations, and the solution to Eq. (4) can be obtained by the simple matrix operation

$$
\begin{aligned}
\vec{\psi}= & {[\overrightarrow{\mathbf{B}}]^{-1} \cdot \overrightarrow{\mathrm{C}}+x[\overrightarrow{\overrightarrow{\mathrm{B}}}]^{-1} \cdot \overrightarrow{\mathrm{D}} } \\
= & \text { function of } \mathrm{T}_{e} \text { and } \mathrm{N}_{e} \\
& +\chi \cdot \text { function of } \mathrm{T}_{\mathrm{e}} \text { and } \mathrm{N}_{\mathrm{e}}
\end{aligned}
$$

Thus the populations of all electronic states are uniquely determined once the macroscopic flow quantities $\mathrm{T}_{e}, \mathrm{~N}_{e}$ and the degree of nonequllibrium $X$ (or equivalently $\mathrm{N}_{\mathrm{a}}$ because $\mathrm{X}$ is related to $\mathrm{N}_{\mathrm{a}}$ through Eq. (7)) are specified. The condition under which the last term in Eq. (5) can be neglected is normally referred to as the quasi-steady condition for the electronic state distribution 4 . Based on the available information on the rate parameter $s^{6,7}$, one can show that such quasi-steady conditions exist in the nozzle flow under consideration. Because the numerical values of the solution, Eq. (8), are known ${ }^{6}$, it is necessary only to find $\mathrm{T}_{e}, \mathrm{~N}_{e}$ and $\mathrm{X}$ (or $\mathrm{N}_{\mathrm{a}}$ ) to determine the nonequilibrium excited state populations.

\section{Flow Equations}

The flow properties $\mathrm{T}_{e}, \mathrm{Ne}_{e}$ and $\mathrm{x}$ are computed by integrating a system of differential equations. The analytical model adopted here is similar to that of Ref. 1 through 3. From Ref. 8, one can write the conservation equations as follows:

(overall continuity)

$$
\begin{aligned}
\rho \mathrm{u} A & =\dot{\mathrm{m}} \\
\rho \mathrm{u} \frac{\mathrm{du}}{\mathrm{d}-} & =-\frac{\mathrm{dp}}{\mathrm{dx}} \\
\rho \mathrm{du} & \frac{\mathrm{dH}}{\mathrm{dx}}=- \text { Qrad }
\end{aligned}
$$

(electron energy)

$$
\begin{aligned}
& N_{e} u \frac{d}{d x}\left[\frac{5}{2} k T_{e}\right]=u \frac{d}{d x}\left(N_{e} k T_{e}\right) \\
& +2 \mathrm{~N} \frac{\mathrm{m}_{\boldsymbol{e}}}{\mathrm{m}_{.}} \nu \mathrm{eh} \underset{\mathrm{z}}{\mathbf{z}}\left(\mathrm{T}_{\mathrm{h}}-\mathrm{T}_{\mathrm{e}}\right)
\end{aligned}
$$

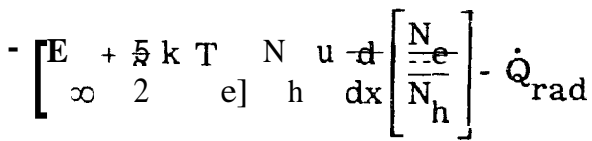

(electron continuity)

$$
\mathrm{N}_{h} \mathrm{u} \frac{\mathrm{d}}{\mathrm{dx}}\left[\frac{\mathrm{N}_{\mathrm{e}}}{\mathrm{N}_{\mathrm{h}}}\right]=\dot{\mathrm{N}}_{\mathrm{e}, \mathrm{rad}}+\dot{\mathrm{N}}_{\mathrm{e}, \text { coll }}
$$

Here, $\mathbf{A}=\mathrm{A}(\mathrm{x})$ is the cross-sectional area of the mnotin. in tho mace flnu rate through the nozzle. 
$\mathbf{p}$ the pressure given by $\mathbf{p}=\mathbf{N}_{\mathbf{e}} \mathbf{k} \mathrm{T}_{\mathrm{e}}+\mathrm{N}_{\mathrm{h}} \mathbf{k} \mathbf{T}_{\mathrm{h}}$, and $\mathbf{H}$ is the total enthalpy per unit mass of plasma

$$
H=\frac{1}{\rho}\left\{\frac{5}{2} k T_{h}+\frac{5}{2} k T_{e}+N_{e} E_{\infty}\right\}+\frac{u^{2}}{2}
$$

In Eq. (14), the energy contained in the excited states $\left[\sum \mathbf{N}(\mathbf{i}) \mathbf{E}(\mathbf{i}) / \rho\right]$ is neglected as being small compared with other forms of energy. The radiation power loss per unit volume Qrad is assumed to be given by

$$
\dot{Q}_{\text {rad }}=\alpha_{r} \mathrm{~N}^{2} \mathrm{E}_{\infty}
$$

where $\alpha_{\mathbf{r}}$ is the overall radiative recombination coefficient for free-bound electron-ion recombination. The radiative recombination coefficient used for nitrogen was

$$
\alpha_{\mathbf{r}} \frac{1.76 \times 10^{-10}}{\mathrm{~T}_{e}^{0.71}} \mathrm{~cm}^{3} \mathrm{sec}^{-1}
$$

which îts the value for nitrogen given at $250^{\circ} \mathrm{K}$ by Bates et al ${ }^{7}$. The temperature dependence was chosen to be the same as that of hydrogen'. When a similar procedire is adopted for hydrogen, the resulting radiative power loss of the form, Eq. (15), is found to give the correct values to within a factor of order 2 as compared to the exact calcu1 a t i o $n$ in which the detailed radiative transport of optically thick lines is included. Thus, while the mechanism of the actual radiation loss is not due only to a n optically thin continuum, the simple expressions, Eq. (15) and (16), are used throughout although a more detailed calculation is possible.

The electron-heavy particle collision frequency is given by

$$
\nu_{\mathrm{eh}}=\overline{\mathrm{v}_{\mathrm{e}}}\left(\mathrm{N}_{\mathrm{a}} \overline{\mathrm{Q}_{\mathrm{ea}}}+\mathrm{N}_{\mathrm{e}} \overline{\mathrm{Q}_{\mathrm{ei}}}\right)
$$

where $\overline{v_{e}}=\sqrt{8 \mathrm{kT}} \bar{e} /\left(\frac{\left.\pi m_{e}\right)}{\mathrm{Q}_{e}}\right.$ is the mean electron thermal velocity. $\bar{Q}_{e a}$ is the electron-atom elastic collision cross-section which is taken from Ref. 10 as $5 \times 10^{-16} \mathrm{~cm}^{2} . \overline{Q_{e i}}$ is the collision cross-section for ion-electron elastic energy exchange, suitably averaged over a Maxwellian velocity distribution, which is taken from Ref. 11.

$\dot{\mathrm{N}}_{\mathrm{e}}, \mathrm{rad}$ is the net radiative rate of electron recombination per unit volume, given by

$$
\mathrm{N}_{\mathrm{e}, \mathrm{rad}}=-\alpha_{\mathrm{r}} \mathrm{N}_{\mathrm{e}}^{2}
$$

Since the plasma is optically thin, photo-ionization has been neglected. The collisional electron-ion reaction rate $\mathrm{Ne}$, coll is obtained from Ref. 12 as

$$
\mathrm{N}_{\mathbf{e}, \text { coll }}=\mathrm{k}_{\mathrm{R}} \mathrm{N}_{\mathrm{e}}^{\mathbf{3}}(\mathrm{x}-1)
$$

where $\mathrm{X}$ is the nonequilibrium parameter defined by Eq. (7). Generally the right-hand-side (RHS) of Eq. (13) cannot be separated into the sum of separate overall radiative and collisional rates 4 , but for the optically thin case with quasi-steady excited state distribution, such a separation is valid 12 . One should note that since most atoms are still found in the ground state, the ground state number density $\mathrm{N}(\mathbf{1})$ is approximately equal to the atom density and hence $\psi(1)=\mathrm{N}(1) / \mathrm{N}_{\mathbf{E}}(\mathbf{1}) \simeq \mathrm{X}$, except at very high temperatures, e. g. $\mathrm{T}_{\mathrm{e}}>25,000^{\circ} \mathrm{K}$. Thus the present analysis is quite similar to that of Ref. 4 in which $\psi(1)$ takes the role of $x$. The col lisional recombination rate coefficient $k_{R}$ was calculated in Ref. 12 for nitroyen as

$$
\mathbf{k}_{\mathbf{R}}=1.15 \times 10^{-26}\left(\mathrm{~T}_{\mathrm{e}} / 10,000\right)^{-5.27} \mathrm{~cm}^{6} \mathrm{sec}^{-1}
$$

which was also verified experimentally ${ }^{13}$. The nitrogen atom and ion partition functions $\mathbf{Z}_{\mathbf{a}}$ and $\mathrm{Z}_{+}$ were evaluated from the tables given by Drellishak 14 through an empirical curve fit.

The basic flow equations (Eq. (9) to (13))are first made dimensionless by dividing by appropriate characteristic values. The equations are then transfornied into a form suitable for machine computation. The details of these steps are similar to those in Ref. 1 and 2. The conservation equations can be written in two forms, an area-specified form 1 or pressure-specified form ${ }^{2}$. The examination of the area-specified form shows two numerical difficulties. First the nonequilibrium solution cannot be started from rest where $\mathrm{u}=0$, because the slopes of the varia ${ }^{1} l e s$ become indeterminate. To overcome this difficulty we initially solve the nozzle flow equations assuming CTE (complete thermodynamic equilibrium) in the very early part of the nozzle flowl using temperature as the independent variable. After the equilibrium solution has yielded a îinite velocity, these equilibrium values are introduced as the initial condition for the nonequilibrium equations. The switch over occurs at a temperature $100^{\circ} \mathrm{K}$ below the chamber temperature.

The second difficulty in the area-specified form lies in the critical point singularity which is often referred to as a saddle point 15 . At the saddlepcint, the denominator of a term in a differential equation becomes zero. Physically the numerator at this point is also zero and so there is no real difficulty, but the coinputational difficulty remains. The saddle point singularity is removed only by adopting the prtssure-specified form2. In the pressurespecified form, only the starting singularity exists which can be overcome as described above. The pressure-specified form has a disadvantage, however, because in most cases the pressure distribution is not known a priori; it is the are a change that is known, at least approximately, rather than the pressure distribution. Thus, a compromise is necessary between the two forms. 
In the present work, the nonequilibrium solution was initiated specifying the pressure distribution and calculating the resulting nozzle profile. After passage of the saddle point, the nonequilibrium solution was continued by solving the areaspecified version. At the switch point between the pressure-specified and area-specified versions, the nozzle contour and slope were made continuous. The pressure distribution assumed for points upstream of the switch pcint was taken to be

$$
\mathrm{p} / \mathrm{p}_{\text {chamb }}=\exp \left[-\left(\mathrm{b}_{2} \mathrm{x}\right)^{\mathrm{s}} .\right.
$$

where the parameters $b_{2}$ and $s$ were found by trial and error so as to generate a nozzle having afixed chamber-to-throat distance of $3.0 \mathrm{~cm}$. The initial area ratio downstream of the switch point was a hyperbolic contour

$$
\mathrm{A} / \mathrm{A}_{*}=\mathrm{I}+\mathrm{b}_{3}\left(\mathrm{x}-\mathrm{x}_{*}\right)^{\mathbf{2}}
$$

where $x_{*}$ is the value of $\mathbf{x}$ at throat. For values of $\mathbf{x}-\mathrm{x}_{*}$ larger than $9.15 \mathrm{~cm}$, this contour was joined smoothly to a parabolic shape in which the nozzle radius was described by

$$
r=b_{4}\left(x-x_{*}\right)^{2}+b_{5}\left(x-x_{*}\right)+b_{6}
$$

where the parameters were so chosen to give zero slope at $\mathbf{x}=18.3 \mathrm{~cm}$ at which point the area ratio became $\approx 22$ if the initial parameters $b_{2}$ and $s$ were appropriately chosen. The maximum half angle of divergence of this nozzle was $11.6^{\circ}$ which occurred at the junction between the hyperbolic and parabolic shapes. A constant area was assumed downstream of the $18.3 \mathrm{~cm}$ station. The mass flow was adjusted to give a throat diameter of $1.27 \mathrm{~cm}$. The resulting nozzle shape is shown in Fig. 1.

\section{Numerical Solution}

The dimensionless form of the flow equations, together with the equilibrium starting solution was programmed for solution on the IBM 360/67 computer. The standard Rungc-Kutta or Adams Moulton methods for integrating a set of simultaneous differential equations proved unsuitable for the solution of these equations except at low pressures, i. e. below 0.1 atm chamber pressure. In the early part of the nozzle flow, very large numerical instabilities are encountered which require a vanishingly small step size. This is commonly referred to as "stiffness"15. Far downstream in the parabolic and constant area sections, however, the standard Adams-Moulton method was satisfactory.

The numerical difficulty arises in the present problem for two reasons. First, the chemical relaxation time associated with the third term in the RHS of Eq. (12) and the second term in the RHS of
Eq. (13) and the thermal equilibration time (second term in IiHS of Eq. (12)) is much smaller than the flow transit time; that is, the chemical and thermalization Damkohler numbers are both much greater than unity. The ratio of the largest to smallest eigenvalues which is a measure of the stiffness was typically of the order of $10^{8}$ for a chamber pressure of $100 \mathrm{~atm}$ and greater for higher pressures. This difficulty is the same as that encountered in the problem of other chemical relaxations such as vibrational 16 or molecular recombination ${ }^{17}$ relaxations, except that the rates associated with plasmas are typically several orders of magnitude greater and hence more "stiff".

Secondly, the elastic thermal equilibration and the ionization relaxation are opposing processes,. which is unique to the relaxing plasma. In Eq. (12), the second and the third terms on the RHS oppose each other in determining $\mathbf{T}_{\mathbf{e}}$. The second term represents the effect of thermalization and tends to lower the electron temperature; the third term signifies that in recombination the ionization energy is given solely to the electron gas and tends to raise its temperature. For instance, let us imagine that the rate of recombination is increased resulting in a closer approach to chemical equilibrium. Because the degree of ionization always decreases in an expanding nozzle, such an increase in the recombination rate leads to a reduction in the slope (i. e. less negative), $\mathrm{dT}_{\mathrm{e}} / \mathrm{dx}$, driving $\mathrm{T}_{\mathrm{e}}$ away from $T_{h}$. In an extreme case, the equilibration process in one mode forces the other mode to deviate further away from equilibrium, resulting in positive values of the normally negative characteristic eigenvalues of the differential equations (see appendix).

In order to obtain satisfactory solutions with reasonably large step sizes, an "implicit" integrating scheme 15 was required with double precision for pressures above $100 \mathrm{~atm}$. The implicit integrating scheme results in a solution which oscillates slightly, the local mean value of which is considered to be the correct solution.

\section{Result and Discussion}

\section{Flow Property Variation}

Because the bound state populations are controlled entirely by the flow quantities $\mathrm{T}_{e}, \mathrm{~N}_{e}$ and the nonequilibrium parameter $X$, the variation of these flow properties is examined first. Table I compares values of various parameters of interest for the following flow models for a representative case:

(a) An ideal frozen flow calculated using the adiabatic isentropic perfect gas equations with $\gamma=1.667$. 
(b) A hypothetical CTE expansion satisfying the Saha equation everywhere with $T_{e}=T_{h}$, including the recombination energy but disregarding radiative losses.

(c) A nonequilibrium expansion satisfying the complete nonequilibrium equations as described in Flow Equations.

(d) Same as (c) except that the radiative loss terms in Eq. (11), (12), and (13), were arbitrarily reduced by a factor of $\mathbf{1 0}$.

The chamber conditions for each model were $\mathrm{p}=10 \mathrm{~atm}$ and $\mathrm{T}=14,000^{\circ} \mathrm{K}$, the nozzle shape being the one described in Flow Equations and shown in Fig. 1.

As seen in Table I, the nonequilibrium solutions (c) and (d) differ greatly from either the frozen flow(a) or equilibrium flow (b) solutions, indicating the need for a nonequilibrium calculation. Nevertheless, the pressure distribution and heavy particle density are not affected greatly by which model is assumed.

Comparison of cases (c) and (d) reveals some interesting effects of radiation. Decreasing the radiation increases both the electron and heavy particle temperature due to decreased radiative cooling. The lower temperature in case (c) allows a higher recombination rate and hence lower values of $\mathrm{Ne}_{e}$. For this case note that this effect has come about because the radiative cooling effect has a larger effectindecreasing $T_{e}$ than the increased recombination rate has in increasing $\mathrm{T}_{e}$. At the exit the value of $X$ in case (d) is nearer to unity primarily because $\mathrm{T}_{e}$ is larger in (d); the exponential dependence of $\chi$ upon $\mathrm{T}_{e}$ has outweighed the square dependence upon Ne.

To illustrate the numerical results, the cases (b) and (c) are exhibited in Fig. 1 and 2. Figure 1 shows at the bottom the nozzle contour used for all the results reported here. In the top of Fig. 1 the values of $T_{e}$ and $T_{h}$ for the nonequilibrium case (c) are compared with the equilihrium temperature TEQ of case (b). Figure 2 shows $\mathrm{N}_{h}, \mathrm{~N}_{e}$ and the parameter $\mathrm{X}$ obtained from two solutions. By definition, $\mathbf{X}=\mathbf{1}$ in the equilibrium flow and so $X$ is shown only for the nonequilibrium case. In this example $X$ reaches $2.37 \times \mathbf{1 0}^{-6}$ at the nozzle exit indicating a significant deviation from equilibrium.

\section{Excited State Populations}

To illustrate the interrelation between the flow quantities $\mathrm{T}_{e}, \mathrm{~N}_{e}$ and $X$ and the electronic state populations, we show a schematic excited state Boltzmannplot in Fig. 3 in which the value of $\log [\mathrm{N}(\mathrm{i}) / \mathrm{g}(\mathrm{i})]$ is shown on an energy level diagram. If all the excited states were in collisional equilibrium with the free electrons, then the resulting CTE value of $\log \left[\mathrm{N}_{\mathrm{E}}(\mathrm{i}) / \mathrm{g}(\mathrm{i})\right]$ would lie along the straight line having slope $-1 / \mathrm{kT}_{\mathrm{e}}$. In the recombining nonequilibrium case, the actual values of $\mathrm{N}(\mathrm{i}) / \mathrm{g}(\mathrm{i})=\psi(\mathrm{i}) \mathrm{NE}_{\mathrm{E}}(\mathrm{i}) / \mathrm{g}(\mathrm{i})$ are smaller than the equilibrium value because $\psi(\mathrm{i})<\mathbf{1}$. As one approaches the ionization limit, however, collisional recombination-ionization processes with the free electrons dominate the excited state transitions and $\psi(\mathrm{i})-1$ as $\mathbf{i}-\infty$, i. e. the highly excited states are in Saha-Boltzmann equilibrium with the free electrons. For the limiting case as one approaches the ionization limit the combination of the Saha and Boltzmann equations results in

$$
\frac{N(\infty)}{g(\infty)}=\frac{\mathrm{N}^{2}}{4.83 \times 10^{15} \mathrm{Z}_{+} \mathrm{T}_{\mathrm{e}}^{1.5}}
$$

Only in the limit i - $\infty$ does the slope of the nonequilibrium distribution approach $-1 / \mathrm{k} \mathbf{T}_{\mathbf{e}}$. As mentioned earlier, the nonequilibrıum parameter $\mathrm{X}$ is approximately equal to $\psi(1)=\mathrm{N}(1) / \mathrm{N}_{\mathrm{E}}(1)$, and so the difference between $\log [N(1) / g(1)]$ and $\log$ $\left[N_{E}(1) / g(1)\right]$, or equivalently the difference between $\log [\mathrm{N}(\infty) / \mathrm{g}(\infty)]$ and $\log \left[\mathrm{N}_{\mathrm{E}}(\infty) / \mathrm{g}(\infty)\right]$, approximately equals $\log \chi$. As $X$ decreases, the population distribution must deviate further away from the equilibrium Boltzmann distribution. Thus $X$ is not only a measure of chemical nonequilibrium but a measure of excitation nonequilibrium, so that it may be called a chemical-excitation nonequilibrium parameter.

The distributions of electronic state populations for the cases shown in Fig. 1 and 2 and Table Iare shown in Fig. 4 at three points; in the stagnation chamber, at the throat and at the exit of the nozzle. The plots in Fig. 4 are obtained by applying Eq. (8), the numerical values of which are found in Ref. 6, using the computed values of $\mathrm{T}_{e}, \mathrm{~N}_{e}$ and $\mathrm{x}$. Figure 4a shows the case of nonequilibrium corresponding to the case (c) in Table I, and Fig. $4 \mathrm{~b}$ is for the equilibrium case (b) in Table I. As seen here the nonequilibrium population distribution does not differ greatly from the equilibrium distribution in the early part of the nozzle flow. At the exit of the nozzle, however, the distribution deviates significantly from the equilibrium distribution, and shows the feature exhibited qualitatively in Fig. 3. At the exit, if a spectroscopist were to calculate the total (or ground state) density based on measurements of the excited state densities $\mathrm{N}(\mathrm{i}), \mathrm{N}_{e}$ and $\mathrm{T}_{e}$, extrapolation downward along the line whose slope is $-1 / \mathbf{k} T_{e}$ on a $E(\mathrm{l})$ vs $\log [\mathrm{N}(\mathrm{i}) / \mathrm{g}(\mathrm{i})]$ plot, he would overestimate the total (or ground state) density by a factor of $1 / \mathrm{x}$ which is approximately $0.4 \times 10^{6}$ in this case. On the other hand if a gasdynamicist were to estimate the excited state density from $\mathrm{N}_{\mathrm{a}}$ even knowing the nonequilibrium value of $\mathbf{N}(1)$ or $\mathrm{N}_{\mathrm{a}}$ together with 
$\mathrm{N}_{e}$ and $\mathrm{T}_{e}$, he would underestimate the upper state density $\mathbf{N}(\mathbf{i})$ by a factor $X$. An even more severe discrepancy would result if the flow were assumed to be completely in equilibrium. When the com plete equilibrium is assumed, one imagines the electronic state populations to lie on the straight line through the ground state at siope - 1/k TEQ where TEQ is only $2599^{\circ} \mathrm{K}$ a s compared to $4727^{\circ} \mathrm{K}$ in the nonequilibrium case. The discrepancy in the upper state populations between the nonequ librium and equilibrium in these cases is approximately $2 \times 1018$.

\section{Effect of Chamber Conditions}

As seen in this example, $\mathrm{X}$ is a second order nonequilibrium parameter dependent on the nonequilibrium values of $\mathrm{T}_{e}$ and $\mathrm{N}_{e}$ as well as the atom density $\mathbf{N}_{\mathbf{a}}$ (see Eq. (5) and (7)). Because of the highly nonlinear appearance of $\mathrm{T}_{e}$ in the nor malizing density $N_{a E}$, a relatively small change in $T_{e}$ can produce a very large change in $X$. For example if one asks what is the order of nonlinearity of the function $\exp \left(-\mathrm{E}_{\infty} / \mathrm{k} \mathrm{T} \mathrm{T}_{e}\right)$ for a typical temperature concerned, say $8400^{\circ} \mathrm{K}$, then by matching the slopes of $\exp \left(-E_{\infty} / k T_{e}\right)$ and $T_{e}{ }^{n}$ one finds the order of nonlinearity $\mathrm{n}$ to be approximately $\mathrm{n} \simeq \mathrm{E}_{\infty} / \mathrm{k} \mathrm{Te}_{\mathrm{e}}=\mathbf{2 0}$. Thus the magnitude of the chemical (and excitation) nonequilibrium pa rameter $\boldsymbol{X}$ is controlled implicitly by the degree of thermal nonequilibrium $\left(T_{e}-T_{h}\right) / T_{h}$ to a great extent. For this reason, the degree of thermal nonequilibrium at the end or the nozzle was calculated systematically as a function of the chamber pressure for various chamber temperatures over the range $0.01 \leq \mathrm{p}_{\text {cham }} \leq 1000 \mathrm{~atm}$ and $6000 \leq$ $\mathrm{T}_{\text {cham }} \leq 18,0000 \mathrm{~K}$. The results are shown in Fig. 5. At low pressures, very little recombination energy is available to force $T_{e}$ and $T_{h}$ apart, the result being that $T_{e}$ and $T_{h}$ are close together. As the pressure increases the recombination rate in Eq. (12) initially dominates over the thermalization term, resulting in a relatively large thermal nonequilibrium. This increasing departure from thermal equilibrium in the range .01 to $1 \mathrm{~atm}$ occurs despite the increase in the corresponding Damkohler number for the thermalization process. At very high pressures, the elastic collision term in Eq. (12) becomes most important, dominating the recombination term and forcing the electron and heavy particle temperature back together. This unique feature is a direct consequence of the opposing nature of the chemical and thermalization processes, which leads to the occurrence of a positive eigenvalue in the differential equations (see appendix).

The chemical-excitation nonequilibr ium parameter $\mathbf{X}$ is calculated also over the same range of conditions. The contours of constant $\log x$ at the nozzle exit are shown in Fig. 6 as functions of the stagnation chamber pressure and temperature. For a fixed chamber temperature, say $14,000^{\circ} \mathrm{K}$, increasing the chamber pressure has the expected effect of forcing the excitation nonequilibrium parameter toward unity only for pressures below about $1 \mathrm{~atm}$. Further increase of the chamber pressure results in a rapid decrease of $X$ with no suggestion over the range of conditions examined here, that $X$ can be forced to return to its equilibrium value of unity. Thus, contrary to intuition, increasing the chamber pressure, and hence the Damkohler number, can drive the flow further away from excitation equilibrium at chamber pressures greater than approximately $10 \mathrm{~atm}$. This unforeseen situation is also a result of the two competing effects described earlier. The closest approach to chemical-excitation equilibrium occurs in the chamber pressure range between 1 and $10 \mathrm{~atm}$. However, 'Fig. 5 shows that in this regime the thermal nonequilibrium is the largest. Thus, one sees that the two types of equilibrium (thermal and chemical) and hence excitation equilibrium, cannot be attained simultaneously under any condition in a fixed nozzle similar to the one under consideration regardless of the magnitude of the associated Damkohler number. Indeed, the Damkohler numbers are typically in the order of unity at the chamber pressures below 0.1 atm for both processes, and extend to $\mathbf{1 0}^{\mathbf{1 2}}$ at the highest pressure and temperature computed.

The exceedingly small values of $\chi$ seen in Fig. 6 do not imply any failure of the conservation of total atoms; even at high temperatures only a small number of the atoms are in excited states, and although there may be $10^{52}$ times too many atoms in the upper excited states, as one would expect from an equilibrium expansion, the absolute number is still a small fraction of the total.

\section{Discussion}

It is seen above that the two types of equilib rium rrocesses do not reach equilibrium simultaneously even when the associated Damkohler numbers are very large. It is interesting also to note that the Damkohler numbers associated with the excitation relaxation process a re equally large even though the excitation process is far out of equilibrium as shown above. The Damkohler number for the excitation relaxation of ith electronic state is the ratio of the flow transit time to the time scale of change of the population ${ }^{18}$ of the ith state $\tau(\mathrm{i})$

$$
\frac{1}{\tau(i)}=N_{e}\left\{K(i, \infty)+\sum_{\substack{j=1 \\ j \neq i}}^{\infty} K(i, j)\right\}+\sum_{j=1}^{i-1} A(i, j)
$$

The largest $\tau(\mathrm{i})$ occurs for the ground state $\mathrm{i}=1$ 
which is obviously not in equilibrium. The next slowest process is for the first excited state which is the 3s state for nitrogen (the two low lying states in nitrogen $2 \mathrm{p}^{3}{ }^{2} \mathrm{D}$ and ${ }^{2} \mathrm{P}$ equilibrate very rapidly ${ }^{19}$ with the ground state and therefore need not be considered) which corresponds to the n (principal quantum number) $=\mathbf{2}$ state in hydrogen. Calculation at $\mathrm{x}=4.36 \mathrm{~cm}$ for the $i 4,000^{\circ} \mathrm{K}$, $10 \mathrm{~atm}$ case shows $\tau(2)=2.9 \times 10^{-10} \mathrm{sec}$ while $\tau(1)=1.3 \times 10^{-4} \mathrm{sec}$ for hydrogen. Considering the fact that these values would hold for nitrogen within an order of magnitude, one concludes that all excited states have relaxation times verymuch smaller than the flow transit time. Because the excitation is shown to be always in nonequilibrium at the nozzle exit, the Damkohler number criteria do not apply in excitation relaxation. Implicitly, however, a large Damkohler number for the excitation process ensures the existence of the quasisteady distribution, as assumed in the derivation.

The main features of the plasma flow that cause such an unusual behavior are:

1. The large Damkohler numbers;

2. The opposing nature of the chemical and thermalization processes;

3. The very small electron-heavy particle mass ratio; and

4. The extreme nonliner.r dependence of the recombination race and Saha function on $\mathrm{T}_{\mathrm{e}}$.

We should like to stress inat the very large excitation nonequilibrium has arisen here as a straight-forward consequence of widely accepted physical principles, without introducing special, ad hoc assumptions as to the existence of meca stabie atoms, resonance radiation trapping, etc. 20-23 'Such phenomena will need to be included in a complete theory, but apparently are not required to produce the rather large nonequilibrium excitation as has been experimentally observed in argon $20-24$, ana to a lesser degree, in nitrogen 3,20 . Also we note that here the flow is assumed to start from complete equilibrium. The nonequilibrium is induced as a result of the competing factors in the expansion process. In actual situations, the starting conditions for the nonequilibrium expansion may not be CTE due to such processes as nonequilibrium excitation in passage through an electric arc.

By combining the calculated $x$ and $T_{e}$ values in Fig. 5 and 6 with Eq. (8) or Ref. 6, one can determine whether an absolute population inversion is achieved that would lead to laser action. Between two levels $\mathrm{i}$ and $\mathrm{j}<\mathrm{i}$, stimulated emission is possible provided

$$
\frac{N(i)}{g(i)}>\frac{N(j)}{g(j)}
$$

or

$$
\psi(\mathrm{i})>\psi(\mathrm{j}) \exp \left[(\mathrm{E}(\mathrm{i})-\mathrm{E}(\mathrm{j})) / \mathrm{kT}_{\mathbf{e}}\right]
$$

The most promising levels in nitrogen are $\mathbf{i}=\mathbf{4}$, $j=3$ (Ref. 5). Now $\psi(3) \simeq x$, here and $\psi(4)$ is very nearly a function only of $\mathrm{T}_{\mathrm{e}}$ for the usual case of $\psi(\mathbf{1})<10^{-3}$. Evaluating $\psi(4)$ from Ref. 6 shows that laser action might be expreted if at any point

$$
x \leqslant 4.8 \exp \left(-101800 / \mathrm{T}_{\mathrm{e}}\right)
$$

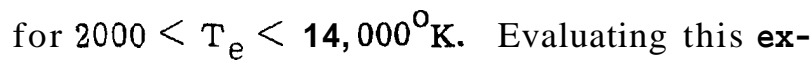
pression for $\mathrm{X}$ using values of $\mathrm{T}_{e}$ at the nozzle exit gives the dotted curve shown on Fig. 6. Laser action appears possible at the nozzle exit for chamber conditions yielding Xexit less than those on the dotted curve.

\section{Conclusions}

A systematic computer study reveals that the three modes of relaxation phenomena in plasma, i. e. thermal, chemical and electronic excitation, are greatly out of equili'srium in a typical wind tunnel nozzle. An extremely large deviation from equilibrium is predicted for the electronic excitation at high pressures. The Damkohler number criteria do not apply to the nonequilibrium plasma processes. The thermal nonequilibrium is largest in the chamber pressure lange between 1 and 10 atm and the chemical excitation nonequilibrium is weakest in the same range.

\section{References}

1. Bray, K.N. C. , "Electron-ion Recombination in Argon Flowing through a Supersonic Nozzle, " High Tempezatu Aspects of Hypersonic Flow, W. Nelson ed., Pergamon Press, 1964, Ch. 4.

2. Talbot, L., Chou, Y.S., and Robben, F., "Expansion of a Partially-Ionized Gas through a Supersonic Nozzle," Report No. AS-65-14, Inst. of Engineering Research, University of California, Berkeley, California, August 1965.

3. Park, C., "Relaxation of Electronic State Populations in Expanding Flows of Ionized Nitrogen, " AIAA preprint No. 68-734, June 1968.

4. Bates, D. R., Kingston, A. E., and McWhirter, R. W. P.. "Recombination between Electrons and Atomic Ions, I. Optically Thin Plasnias," Proc. Roy. Soc., A267, 297 (1962).

5. Park, C., "Theoretical Population Inversion in Decaying Nitrogen Plasma Column," AIAA Preprint No. 69-48, January, 1969. 
6. Park, C., "Spectral Line Intensities in a Nonequilibrium Nitrogen Plasma, "Journ. Quant. Spect. Rad. Transf, 8, 1633 (1968).

7. Bates, D. R. and Dalgarno, A., "Electronic Recombination, "Atomic and Molecular Processes, D.R. Bates, ed., Academic Press, 1962, Ch. 7, pp. 252.

8. Appleton, J.P. and Bray, K.N.C., "The Conservation Equations for a Nonequilibrium Plasma," Journ. Fluid Mech., 20, 659 (1964). !

9. Lasher, L. E., Wilson, K.H., and Grief, R., "Radiation from an Isothermal Hydrogen Plasma at Temperatugre s up to $40,000^{\circ} \mathrm{K}$," Journ. Quant. Spect. Rad. Transf., 7, 305 (1967).

10. Pos, J., "Transport and Thermodynamic Prcperties of Nitrogen, Hydrogen, Oxygen and Air to $30,000^{\circ} \mathrm{K}$, "Appendix A in Theoretical and Experimental Investigation of Arc Plasma Generation Technoloey. Part II. Vol. 2. R.R. John et al. AVCO RAD.

11. Kruger, C.H. and Viegas, J.R., "Influence of the Ramsaur Effect on Nonequilibrium Electron Temperatures," Phys. Fluids, I, 1879 (1964).

12. Park, C. ,"Collisional Ionization and Recombination Rates of Atomic Nitrogen, "AIAA J., 7, 1653 (1969).

13. Park, C., "Measurement of Ionic Recombina tion Rate of Nitrogen, " A I A A J. , 5, 2090 (1968).

14. Drellishak, K.S., Aeschliman, D. P., and Cambel, A. B., "Tables of Thermodynamic Properties of Argon, Nitrogen and Oxygen Plasmas, " Northwestern Univ. Rept. No. AEDC-TDR-64-12, January 1964 .

15. Lomax, H., "Stable Implicit and Explicit Numerical Methods for Integrating QuasiLinear Differential Equations with ParasiticStiff and Parasitic-Saddle Eigenvalues," NASA TN D-4703, July, 1968.

16. Stollery, J. L. and Park, C., ' Computer Solutions to the Problem of Vibrational Relaxation on Hypersonic Nozzle Flows," Journ. Fluid Mech., 19, 113 (1964).

17. Bray, K. N.C., "Atomic Recombination in a Hypersonic Wind-Tunnel Nozzle, " Journ. Fluid Mech., 6, 1 (1959).
18. McWhirter, R.W.P. and Hearn, A.G., "A Calculation of the Instantaneous Population Densities of the Excited Levels of HydrogenIike Ions in a Piasma, .. Proc. Phys. Soc., 82, 641 (1963).

19. Smith, K., Henry, R. J.W., and Burke, P. G., "Calculations on the Scattering of Electrons by Atomic Systems with Configurations 2pq," Phys. Rev., 157, 151 (1967).

20. Staats, G.E. and McGregor, W. K., "Vacuum Ultraviolet Radiation Emitted fronı Arc Jet Plasmas of Argon or Nitrogen, "AEDC-TDP68-171, (1968).

21. Brewer, I.E., Jr., "Spectroscopy of a Supersonic Plasma, I. Excitation Mechanism\%" AEDC-TDR-64-151 (1964), II. Excitation Temperature," AEDC-TDR-64-196 (1964).

22. Brewer, L.E., Jr., "Plasma Radiation Resulting from an Over-Population of Atcms in the Metastable State, "MS thesis, Univ. of Tennessee, March 1965.

23. Bowen, S. W. , "A Spectroscopic Study of an Underexpanded Argon Plasma Jet, " Ph. D. thesis, Univ. of Michigan, Ann Arbor, Michigan, 1966.

24. Bomen, S. W., "Spectroscopic and Optical Studies of a High Pressure, Underexpanded Jet," AIAA Preprint No. 66-164, March 1966. 
Table I. Calculated Flow Parameters Expanding from $\mathbf{p}_{\text {cham }}=\mathbf{1 0} \mathrm{atm} \mathbf{T}_{\text {cham }}=14,000^{\circ} \mathrm{K}$.

a) Ideal Frozen Flow, $\mathrm{m}=30.48 \mathrm{gm} / \mathrm{sec}$

\begin{tabular}{l|ccc}
\multicolumn{1}{c}{} & Chamber & Throat & Exit \\
\cline { 2 - 5 } $\mathbf{T}_{\mathbf{e}}, \mathbf{0}_{\mathbf{K}}$ & 14000 & 10447 & 862 \\
$\mathbf{T}_{\mathrm{h}}, \mathbf{o}_{\mathbf{K}}$ & 14000 & 10447 & 862 \\
p. atm & 10 & 4.811 & $9.421 \times 10^{-3}$ \\
$\mathbf{N}_{\text {tot }} \mathbf{c m}^{-\mathbf{3}}$ & $5,243 \times 10^{18}$ & $3.380 \times 10^{18}$ & $8,018 \times 10^{16}$ \\
$\mathbf{u}, \mathbf{c m} / \mathbf{s e c}$ & 0 & $3.247 \times 10^{5}$ & $6.244 \times 10^{5}$ \\
A. $\mathbf{c m}^{\mathbf{2}}$ & $\infty$ & 1.267 & 27.78 \\
& & &
\end{tabular}

它

c) Nonequilibrium Flow, $m=29.37 \mathrm{grn} / \mathrm{sec}$

\begin{tabular}{|c|c|c|c|c|}
\hline & Chamber & Throat & $\begin{array}{c}\text { Saddle } \\
\text { Point }\end{array}$ & Exit \\
\hline$x_{1},: m$ & 0 & 2.002 & 2. 322 & 24.92 \\
\hline $\mathrm{T}_{e},{ }^{\circ} \mathrm{K}$ & 14000 & 12175 & 11879 & 4247 \\
\hline $\mathrm{T}_{\mathrm{h}},{ }^{\circ} \mathrm{K}$ & 14000 & 12090 & 11744 & 1888 \\
\hline $\mathrm{p}$, atm & 10 & 5. 510 & 4. 708 & $1.581 \times 10^{-2}$ \\
\hline $\mathrm{N}_{\mathrm{t}}, \mathrm{cm}^{-3}$ & $5.807 \times 10^{17}$ & $.882 \times 10^{17}$ & $1.467 \times 10^{17}$ & 1. $742 \times 10^{14}$ \\
\hline$N_{\mathrm{f}}, \mathrm{cm}^{-3}$ & 4. $662 \times 10^{18}$ & 3. $156 \times 10^{18}$ & $2.794 \times 10^{18}$ & $6.102 \times 10^{16}$ \\
\hline$N_{t} / N_{h}$ & .1246 & .05965 &, 05252 & .00285 \\
\hline $\mathrm{u}, \mathrm{cm} / \mathrm{sec}$ & 0 & 3. $136 \times 10^{5}$ & $3.489 \times 10^{5}$ & $7.225 \times 10^{5}$ \\
\hline A. $\mathrm{cm}^{2}$ & $\infty$ & 1.268 & 1.287 & 27.70 \\
\hline$x$ & 1.00 & 1.000 & .9981 & $2.366 \times 10^{-6}$ \\
\hline
\end{tabular}

b) CTE Flow, $\mathrm{m}=\mathbf{2 7 . 6 2} \mathrm{gm} / \mathrm{sec}$

\begin{tabular}{|c|c|c|c|c|}
\hline & Chamber & Throat & $\begin{array}{l}\text { Saddle } \\
\text { Point }\end{array}$ & Exit \\
\hline $\mathrm{x}, \mathrm{cm}$ & 0 & 2.014 & 2.372 & 249 \\
\hline $\mathrm{T}_{\mathrm{e}},{ }^{\mathrm{O}_{\mathrm{K}}}$ & 14000 & 12900 & 12600 & 2599 \\
\hline $\mathbf{T}_{\mathrm{h}},{ }^{\mathbf{o}_{\mathrm{K}}}$ & I4000 & 12900 & 12600 & 2509 \\
\hline $\mathrm{p}$, atm & 10 & 5.479 & 4.589 & $1.888 \times 10^{-2}$ \\
\hline $\mathrm{N}_{\mathrm{e}}, \mathrm{cm}^{-3}$ & $5.807 \times 10^{17}$ & $2.700 \times 10^{17}$ & $2.127 \times 1017$ & 7. $452 \times 10^{4}$ \\
\hline $\mathrm{N}_{\mathrm{h}}, \mathrm{cm}^{-3}$ & 4. $662 \times 1018$ & $2.84^{\prime} 7 \times 10^{18}$ & $2.460 \times 10^{\mathbf{1 8}}$ & $5.330 \times 10^{16}$ \\
\hline $\mathrm{N}_{\mathrm{e}} / \mathrm{N}_{\mathrm{h}}$ & 1246 & .09482 & .08647 & $1.398 \times 10^{-12}$ \\
\hline $\mathrm{u}, \mathrm{cm} / \mathrm{sec}$ & 0 & $3.268 \times 10^{5}$ & $3.690 \times 10^{5}$ & $7.965 \times 10^{5}$ \\
\hline $\mathrm{A}, \mathrm{cm}^{2}$ & $\infty$ & 1.268 & 1.300 & 27.80 \\
\hline$x$ & 1.00 & 1.00 & 1.00 & 1.00 \\
\hline
\end{tabular}

d) Nonequilibrium Flow, radiation decreased by factor 10 $\mathbf{m}=27.74 \mathrm{gm} / \mathrm{sec}$

\begin{tabular}{|c|c|c|c|c|}
\hline & Chamber & Tnroat & $\begin{array}{l}\text { Saddle } \\
\text { Point }\end{array}$ & Exit \\
\hline$x, \operatorname{con}$ & 0 & 1.983 & 2.303 & 24.921 \\
\hline $\mathrm{T}_{\mathrm{e}},{ }^{\mathrm{O}} \mathrm{K}$ & 14000 & 12835 & 12528 & 5476 \\
\hline $\mathrm{T}_{\mathrm{h}},{ }^{\mathrm{o}} \mathrm{K}$ & 14000 & 12778 & 12437 & 2753 \\
\hline $\mathrm{p}$, atm & 10 & 5.508 & 4.583 & $2.000 \times 10^{-2}$ \\
\hline $\mathrm{N}_{\mathrm{e}}, \mathrm{cm}^{-3}$ & $5.807 \times: 0^{17}$ & $2.635 \times 10^{17}$ & $2.059 \times 10^{17}$ & 2. $779 \times 10^{14}$ \\
\hline $\mathrm{N}_{\mathrm{h}}, \mathrm{cm}^{-3}$ & $4.662 \times 10^{18}$ & $2.899 \times 10^{18}$ & $2.497 \times 10^{18}$ & 5. $277 \times 10^{16}$ \\
\hline $\mathrm{Ne} / \mathrm{Nh}_{\mathrm{h}}$ &, 1246 & ,09089 & .08248 & .00527 \\
\hline $\mathrm{u}, \mathrm{cm} / \mathrm{sec}$ & 0 & $3.226 \times 10^{5}$ & $3.658 \times 10^{5}$ & $7.748 \times 10^{5}$ \\
\hline $\mathrm{A}, \mathrm{cm}^{2}$ & $\infty$ & 1.267 & 1.298 & 28.22 \\
\hline$x$ & 1.00 & ,9992 & .9993 & $1.301 \times 10^{-4}$ \\
\hline
\end{tabular}




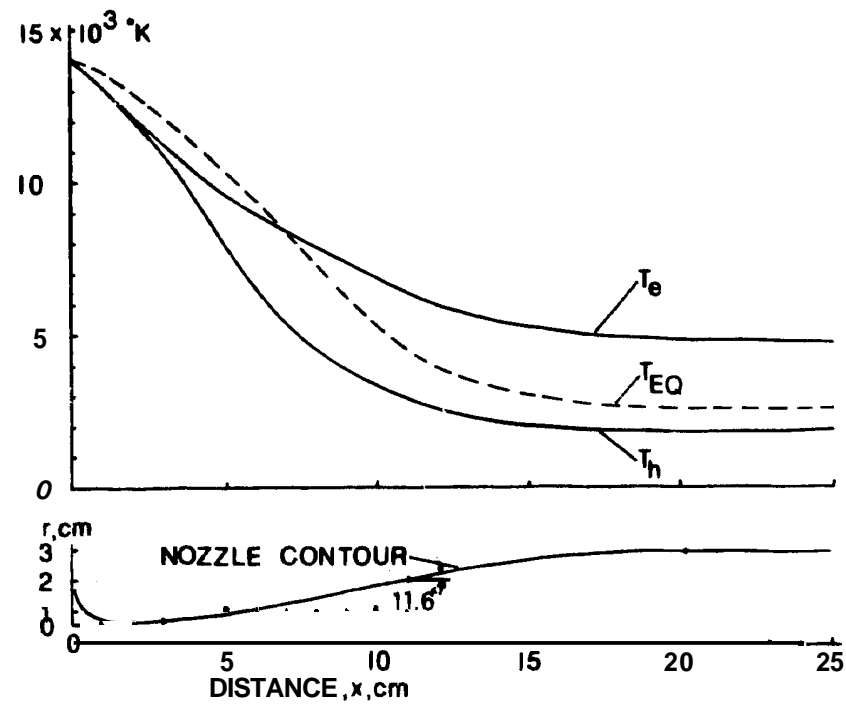

Figure 1. Nozzle Contour and Temperature'Distributions through the nozzle, $A_{\text {exit }} / A_{*}=22$.

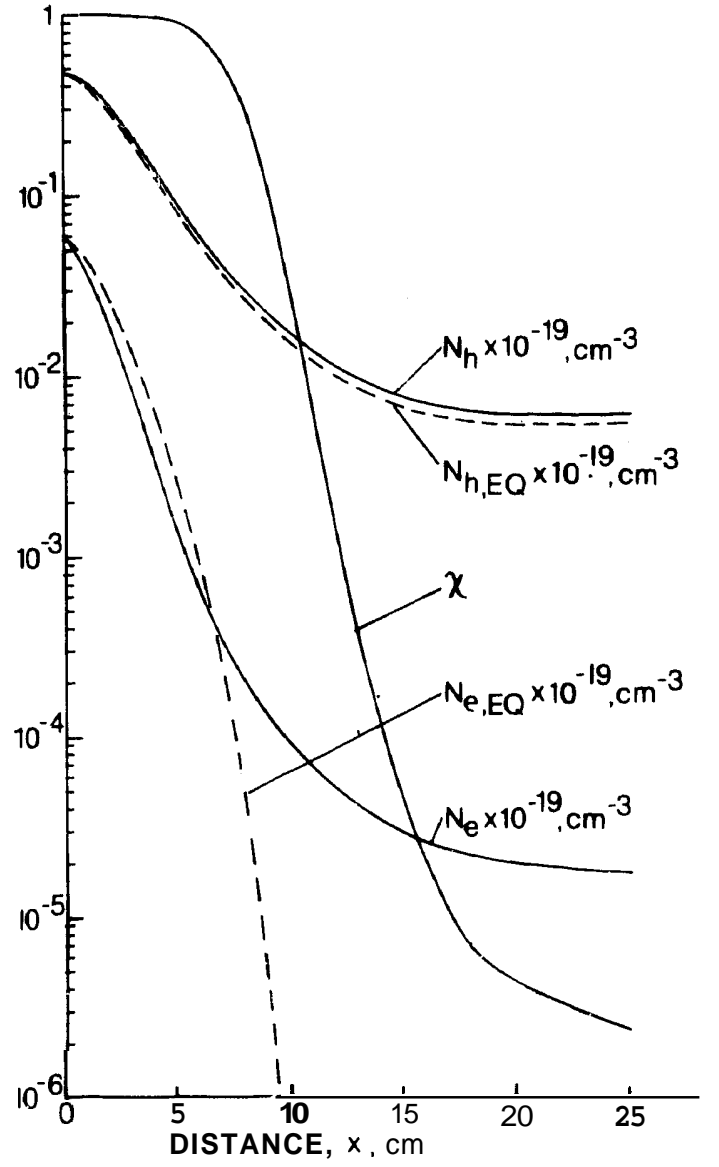

Figure 2. The Variation of Particle Densities and the Nonequilibrium Parameter $x$ through the Nozzle, $A_{\text {exit }} / A_{*}=22$.

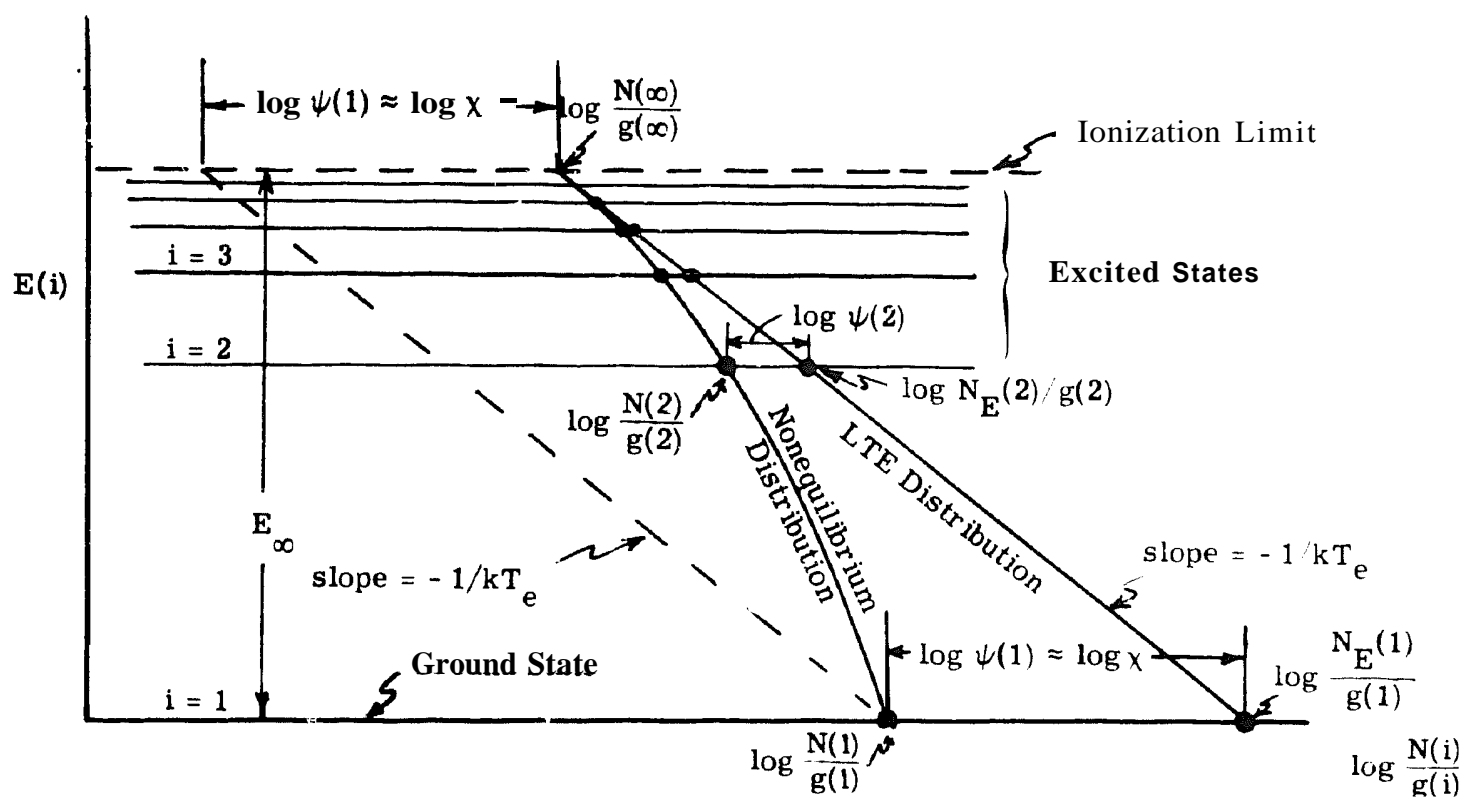

Figure 3. Schematic Excited State Boltzmann Plot for Recombining Flow. 

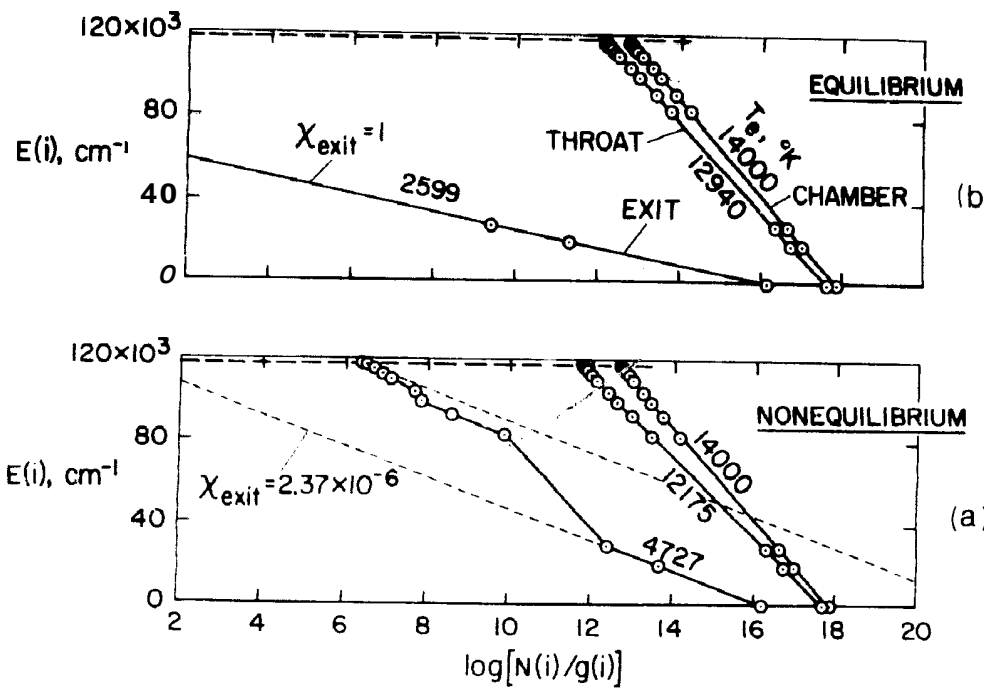

Figure 4. Electronic State Populations in Nitrogen Plasma Nozzle Flows. $\mathrm{A}_{\text {exit }} / \mathrm{A}_{*}=22, \mathrm{~T}_{\text {cham }}=14,000^{\circ} \mathrm{K}, \mathrm{p}_{\text {cham }}=10 \mathrm{~atm}$.

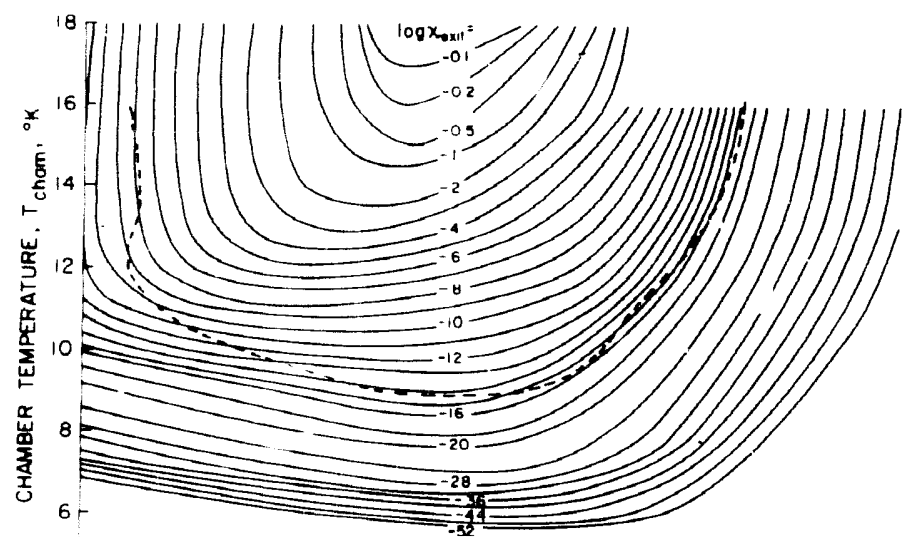

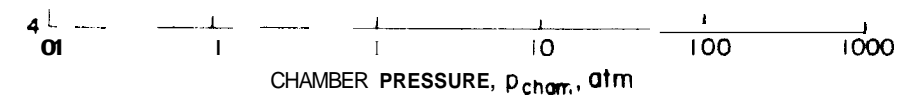

Figure 6. Contours of Constant $\log X_{\text {exit }}$. Dotted curve indicates the boundary of possible population inversion $\mathrm{Aexit} / \mathrm{A} * \mathbf{2 2}$.

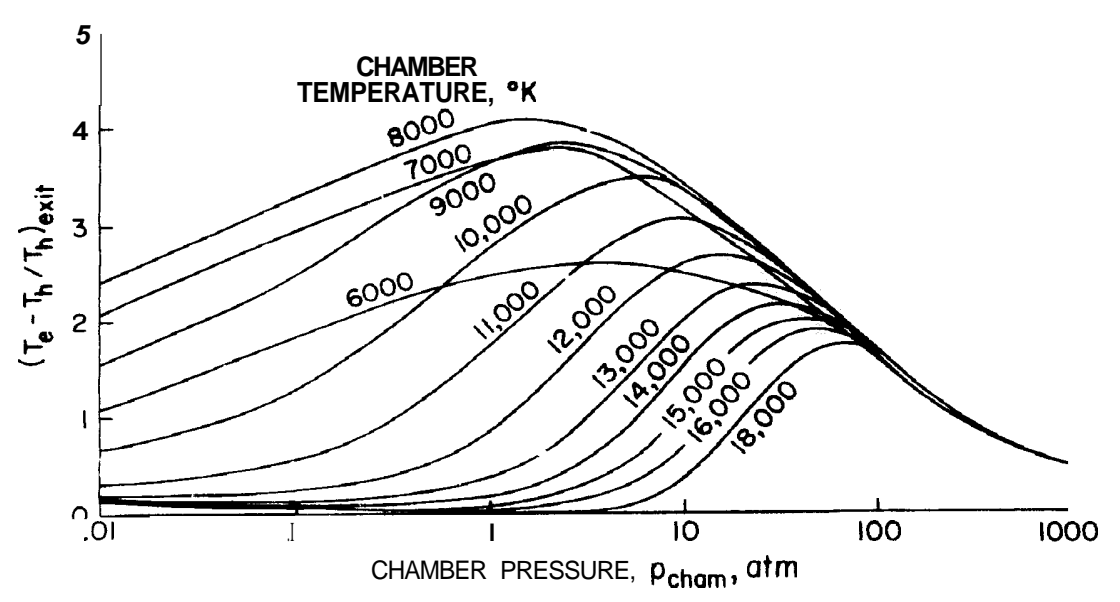

Figure 5. Degree of Thermal. Nonequilibrium at the Nozzle Exit as a Function of the Chamber Pressure for Various Chamber Temperatures, $\mathrm{A}_{\text {exit }} / \mathrm{A}_{*}=22$.

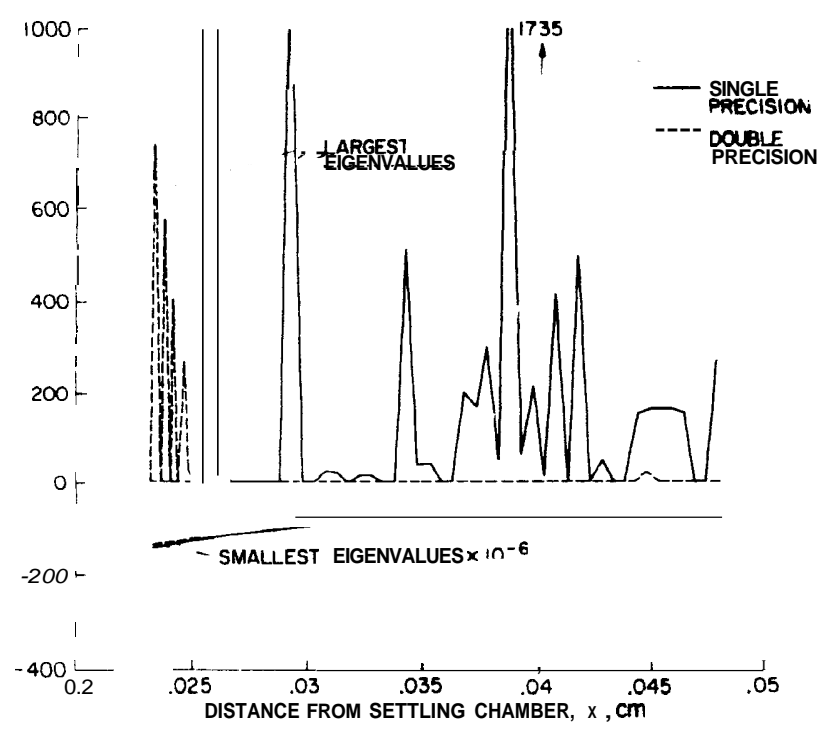

Figure 7. Variation of the Largest and Smallest Eigenvalues Along the Nozzle, $\mathrm{p}_{\text {cham }}=1000 \mathrm{~atm}, \mathrm{~T}_{\text {cham }}=14,000^{\circ} \mathrm{K}$. 


\section{APPENDIX}

Occurrence of a Positive Eigenvalue in a Relaxing Plasma

In most nonequilibrium chemical processes? the relaxing quantities approach equilibrium monotonically in an exponential decay. That is, the change in a relaxing quantity y during a small time interval $\Delta t$ can be described typically by

$$
y-y_{E}=C \exp (-t / \tau)
$$

where $\mathbf{y}_{\mathbf{E}}$ is the equilibrium value of the quantity $y$ and 7 is the characteristic relaxation time. For plasmas, the relaxation of electron temperature and degree of ionization does not necessarily obey this general rule because of the opposing effect of the two relaxation processes. Under a certain condition, the equilibration of one process causes a departure from equilibrium in the other. To illustrate this peculiar behavior mathematically, one can assume a uniform, motionless plasma confined in an adiabatic container, which is in chemical equilibrium but thermal nonequilibrium at time $t=\boldsymbol{0}$. The degree of ionization is so small that the energy content of electron gas is considered not sufficient to affect the heavy particle temperature appreciably during the relaxing period $t>0$. The radiation is neglected altogether because it is not essential in describing the competition between the two processes. " he relaxation process for this plasma for $t>0$ can be described approximately by two simultaneous differential equations

$$
\begin{gathered}
\frac{\mathrm{d}}{\mathrm{dt}}\left\{\alpha\left[\frac{3}{2} \theta_{\mathrm{e}}+1\right]\right\}=3 \frac{\mathrm{m}_{\mathrm{e}}}{\mathrm{m}_{\mathrm{h}}} \nu_{\mathrm{eh}} \alpha\left(\theta-\theta_{\mathrm{e}}\right) \\
\frac{\mathrm{d} \alpha}{\mathrm{dt}}=\mathrm{kR} \mathrm{Nh}^{2} \alpha\left\{(1-\alpha) \frac{{ }_{0} \mathrm{e}_{\eta}}{\eta} \exp \left(-1 / \theta_{\mathrm{e}}\right)-\alpha^{2}\right\}
\end{gathered}
$$

$\eta$ is the densitg normalized by the characteristic of ionizationt, $8, \theta_{\mathrm{e}}$ are heavy particle and electron temperature normalized by $E_{\infty} / \mathrm{k}$.

During the small time span $0<t<A$ t, the above equations can be linearized as follows

$$
\begin{aligned}
& \frac{\mathrm{d} \theta_{\mathrm{e}}}{\mathrm{d} \mathrm{t}}=\mathrm{a}_{11}\left(\theta_{\mathrm{e}}-\theta_{\mathrm{o}}\right)+\mathrm{a}_{12}\left(\alpha-\alpha_{\mathrm{E}^{\prime}}\right) \\
& \frac{\mathrm{d} \alpha}{\mathrm{dt}}=-\mathrm{a}_{21}\left(\theta_{\mathrm{e}}-\theta_{\mathrm{o}}\right)+\mathrm{a}_{22}\left(\alpha \cdot \alpha_{\mathrm{E}}\right)
\end{aligned}
$$

Here the coefficients $a_{i j}$ are

$$
\begin{aligned}
\mathrm{a}_{11} & =-\left(\Gamma_{\mathrm{c}}+\frac{z}{3} \theta_{0}-2 \Gamma_{\mathrm{r}}\right) \\
\mathrm{a}_{12} & =\frac{4}{3} a_{\mathrm{E}}{ }^{-1} \Gamma_{\mathrm{c}} \\
& =\alpha_{\mathrm{E}}{ }^{\theta}{ }_{0}^{-2} \Gamma_{\mathrm{r}}
\end{aligned}
$$

$$
a_{22}=-2 \alpha_{E} \quad r_{r}
$$

The subscripts $\boldsymbol{O}$ and $\mathbf{E}$ refer to $\mathrm{t}=0$ and equilibrium, respectively. In Eq. (A 3), $\Gamma_{\mathrm{C}}$ and $\Gamma_{\mathrm{r}}$ are the rate parameters for collisional (i.e. thermalization) process and chemical reaction, respectively, defined as

$$
\begin{aligned}
\Gamma_{\mathrm{c}} & \equiv 2 \frac{\mathbf{m}_{\mathrm{e}}}{\mathrm{m}_{\mathrm{h}}} \nu_{\mathrm{eh}} \\
\mathrm{r}_{\mathrm{r}} & \equiv \mathrm{k}_{\mathbf{R}} \mathbf{N}_{\mathrm{h}}{ }^{2}
\end{aligned}
$$

and can be considered as the reciprccals of the characteristic times of the processes concerned. In deriving Eq. (A.2), the terms of the order $\theta_{\mathbf{e}}$ and $\alpha$ are neglected in comparison with unity. The general solution to Eq. (A2) is

$$
\begin{aligned}
& \theta_{\mathrm{e}}-\theta_{\mathrm{o}}=\mathrm{C}_{1} \exp \left(\lambda_{1 \mathrm{t}}\right)+\mathrm{C}_{2} \exp \left(\lambda_{2 \mathrm{t}}\right) \\
& \alpha-\alpha_{\mathrm{E}}=\mathrm{C}_{3} \exp \left(\lambda_{1 \mathrm{t}}\right)+\mathrm{C}_{4} \exp \left(\lambda_{2 \mathrm{t}}\right)
\end{aligned}
$$

where $\lambda_{1}$ and $\lambda_{2}$ are the eigenvalues of the matrix $a_{i j}$ of Eq. (A2)

$$
\begin{gathered}
\lambda_{1}=\frac{1}{2} \mid a_{11}+a_{22} \\
\left.\quad-\sqrt{\left(a_{11}+a_{22}\right)^{2}-4\left(a_{11} a_{22}-a_{21} a_{12}\right)}\right] \\
\lambda_{2}=\frac{1}{2}\left[a_{11}+a_{22}\right. \\
\left.+\sqrt{\left(a_{11}+a_{22}\right)^{2}-4\left(a_{11} a_{22}-a_{21} a_{12}\right)}\right]
\end{gathered}
$$

On substitution of Eq. (A.3) into (A5), one finds that the two eigenvalues $\lambda_{1}$ and $\lambda_{2}$ are always real, and that they can have the following two sets:

(a) both are negative if $r_{\mathrm{c}}<\alpha \Gamma_{\mathrm{r}}$

(b) $\lambda_{2}$ becomes positive while $\lambda_{1}$ is negativeif IC $>\alpha I_{r}$

In case (a), both thermalization and chemical relaxation processes approach equilibrium exponentially with time as in ordinary chemical processes, e.g. Eq. (Al). That is, when the chemical equilibration rate is faster than the thermalization rate, the rlasma relaxes into equilibrium monotonically €or both processes. In case (b), $1 . e$. when the thermalization process is dominant over the chemical relaxation process, there is a component in $\mathrm{Eq}$. (A4) that tends to drive a relaxing quantity away from equilibrium. Depending on the initial condition, this component containing the positive eigenvalue may become apparent in thr overall solution, thereby resulting in a departure from equilibrium.

The step size in numerical integration must be reduced inversely with the magnitude of the nncitive eirrenvelue and thereforn a larege pecitim 
eigenvalue results in a long computation time.

In both cases (a) and (b) above, a large numerical error can be introduced in evaluating $\lambda_{2}$ if $\left|\mathbf{a}_{11} \mathbf{a}_{22}-\mathbf{a}_{21} \mathbf{a}_{12}\right|$ is small. As seen from Eq. (A5), when $\left|\mathbf{a}_{11} \mathbf{a}_{22}-\mathbf{a}_{21} \mathbf{a}_{12}\right|$ is small, $\lambda_{2}$ is a difference between two almost identical numbers. An example of this case is shown in Fig. 7, which shows the variation in eigenvalues quite early in the expansion for one of the cases computed in the present study. The case considered corresponds to the chamber temperature and pressure of $14,000^{\circ} \mathrm{K}$ and $1,000 \mathrm{~atm}$. The figure shows the smallest, i. e. most negative, eigenvalue, which corresponds to $\lambda_{\mathbf{1}}$ in Eq. (A5). The full-lined curves are obtained with single precision computation by I3M 360/67 while the dotted curves are from the double precision calculations. As seen in the figure, the smallest eigenvalues are the same between the single and double precision calculations, but the largest eigenvalues are quite different between the two computations, thereby indicating the sensitivity of the eigenvalue on the degree of precision. 